\title{
Relaciones evolutivas entre la memoria de trabajo visuoespacial y la planificación cognitiva en personas sanas con inteligencia normal con edades entre 10 y 30 años*
}

\section{Developmental relationships between visuospatial working memory and cognitive planning in healthy people with normal intelligence aged between 10 and 30 years}

\author{
Jorge Emiro Restrepo** \\ Tecnológico de Antioquia Institución \\ Universitaria \\ María José Puello \\ Jenny Shirley Ramírez \\ Julia Johana Rivas \\ Jennifer Teresa Romero \\ Universidad Cooperativa de Colombia \\ Recibido: 9 de noviembre de 2016 \\ Revisado: 25 de enero de 2017 \\ Aceptado: 3 de abril de 2017
}

\section{Resumen}

El artículo presenta los resultados de una investigación empírica sobre las relaciones evolutivas entre la memoria de trabajo visuoespacial y la planificación cognitiva en un grupo de 100 personas sanas con inteligencia normal y edades entre 10 y 30 años. Se aplicó el Subtest de Cubos de Corsi, de la Escala de Memoria de Wechsler III y la Torre de Londres. Los análisis de correlación para el rango completo (10-30) demostraron la existencia de una correlación estadísticamente significativa $(0.43 ; p$-value $<0.001)$. En los tres rangos de edad también se encontraron correlaciones estadísticamente significativas ( $p$ value < 0.008): 0.44 para el rango 10-15; 0.28 para el rango 16-20; y 0.32 para el rango 21-30. No se encontró un aumento en el funcionamiento ejecutivo asociado con la edad, pese a que la teoría y la evidencia empírica así lo demuestran. Se describen y discuten posibles explicaciones sobre los resultados.

Palabras clave: memoria de trabajo visuoespacial, planificación cognitiva, desarrollo cognitivo.

* $\quad$ Artículo de investigación. DOI: http://dx.doi.org/10.15332/s1794-9998.2017.0002.07

** Correspondencia: Jorge Emiro Restrepo, Facultad de Educación y Ciencias Sociales, Tecnológico de Antioquia Institución Universitaria. Medellín, Antioquía. Dirección postal: calle 78B No. 72A-220; Laboratorio de Psicología (1- 302); Tecnológico de Antioquia, Medellín, Colombia. Correo electrónico: jorge.restrepo67@tdea.edu.co 


\section{Abstract}

The article presents the results of a correlational empirical research on the developmental relationships between visuospatial working memory and cognitive planning in a group of 100 healthy people with normal intelligence with ages between 10 and 30 years. The Corsi block test, from the Wechsler Memory Scale III, and the Tower of London were applied. Correlation analyzes for the full range (10-30) demonstrated the existence of a statistically significant correlation (0.43, p-value $<0.001$ ). In the three age statistically significant correlations ( $p$ value $<0.008$ ) were also found: 0.44 for the range $10-15 ; 0.28$ for the range $16-20$; and 0.32 for $21-30$ range. No increase in executive functioning associated with age was found, although the theory and empirical evidence prove it. Possible explanations for the results are described and discussed.

Keywords: visuospatial working memory, cognitive planning, cognitive development.

\section{Introducción}

La memoria de trabajo visuoespacial y la planificación cognitiva son funciones ejecutivas asociadas anatómicamente con la corteza prefrontal. Ambas forman parte de las funciones ejecutivas. Estas tienen como principal objetivo la adaptación al contexto a partir de la preparación, ejecución, regulación y ajuste del comportamiento, la emoción y la cognición. La memoria de trabajo visuoespacial forma parte de la memoria de trabajo, en general, y de la agenda visuoespacial, en particular. La función de este tipo especial de memoria es el mantenimiento, en la memoria de trabajo, de una imagen visual del estímulo.

Este mantenimiento temporalmente breve tiene como objetivo permitirle al sistema cognitivo, direccionado por el ejecutivo central, mantener una línea de ejecución sintonizada entre las condiciones externas e internas, a partir de representaciones rápidas que permanecen on-line el tiempo suficiente para que el sistema pueda preparar, ejecutar, regular o ajustar su dinámica. La planificación cognitiva es la función ejecutiva que le permite al sistema organizar su funcionamiento en línea con las demandas ambientales. La planificación establece un orden de ejecución que sea satisfactorio para los recursos y las exigencias. Este tipo de función parece ser una función de segundo orden en la jerarquía de las funciones ejecutivas.
Aunque hay relativa claridad y consenso en los componentes de la función ejecutiva, hasta ahora ha sido difícil definir si todos ellos, con independencia de cuántos sean, tienen una organización horizontal, sin jerarquía, o si, por el contrario, responden a alguna forma vertical, jerárquica, en la que algunos de sus componentes son requisitos funcionales para los demás. La memoria de trabajo visuoespacial es un tipo de función que requiere de mecanismos sensoriales y perceptuales (representacionales) no necesariamente ejecutivos, aunque en sí misma sea considerada como ejecutiva. Más allá de qué tanta sensación o percepción (no ejecutiva) haya en la memoria de trabajo visuoespacial, la planificación cognitiva parecería requerir de otros mecanismos de bajo nivel (jerarquía vertical) tanto ejecutivos como no ejecutivos (Luciana, Collins, Olson, Schissel, 2009).

La hipótesis que ha motivado esta investigación parte de la suposición de que la planificación cognitiva, como función ejecutiva, involucra la participación de otros mecanismos ejecutivos y no ejecutivos. Uno de estos mecanismos estaría asociado con la memoria de trabajo visuoespacial, bien directamente con toda la función, o bien parcialmente con algún mecanismo particular de esta función. Esta hipótesis parte de inferencias teóricamente plausibles y de evidencia empírica de algunos estudios que han analizado esta asociación entre la memoria de trabajo visuoespacial y 
la planificación cognitiva. Las inferencias teóricas parten de la idea de que la planificación requiere la generación de modelos mentales o representaciones anticipadas que permitan generar un plan a partir del cual se pueda realizar una anticipación cognitiva de la acción.

Por ejemplo, es plausible inferir que, en la ejecución de la Torre de Londres (una tarea típica para evaluar la planificación cognitiva según los protocolos neuropsicológicos), es necesaria la participación de un componente de memoria visuoespacial. Durante la ejecución, el evaluado debe mantener en su memoria, con dos propósitos, los últimos movimientos realizados: primero, para no repetir aquella secuencia de movimientos que no haya resultado efectiva, y, segundo, para establecer cuál es la secuencia de movimientos qué ya ha resultado satisfactoria. Además de los otros mecanismos que requiera la planificación, es plausible considerar que el mantenimiento on-line de los últimos movimientos realizados sean un elemento necesario para tomar decisiones sobre los movimientos que deberán realizarse (planificación) ejecutivos (Luciana et al., 2009).

Phillips, Wynn, Gilhooly, Della Sala, y Logie (1999) ya habían sugerido que la memoria de trabajo hacía parte de las funciones asociadas con la planificación cognitiva, tal y como se evalúa con la Torre de Londres (TOL). Según ellos, "en la TOL, parece plausible que el ajuste sobre la marcha, el mantenimiento y la ejecución de un plan de múltiples etapas hará que se exija mucho trabajo sobre los recursos de memoria" (p.211). Cohen (1996) también sostenía que "la memoria de trabajo es importante en la formulación, la retención, y la implementación de planes, así como la revisión de las mismas en línea" (Phillips, Wynn, Gilhooly, Della Sala y Logie, 1999, p.211).

Phillips et al., (1999) argumentan que los requerimientos de presentación y de respuesta de la TOL son visuales y espaciales, pero no se ha clarificado si la planificación, en sí misma, requiere del uso de recursos de memoria verbal o visuoespacial. Estos autores se preguntan si las personas resuelven la tarea mediante la verbalización silente (subvocalización) de un plan de acción, tal y como lo sugieren Morris, Ahmed, Syed, y Toone (1993), o si la resolución de la tarea ocurre mediante la visualización de los movimientos que se realizan durante la ejecución, tal y como se ha reportado en otros estudios (Joyce y Robbins, 1991; Owen, Downes, Sahakian, Polkey, y Robbins, 1990; Welsh, Cicerello, Cuneo, y Brennan, 1995).

Temple, Carney, y Mullarkey, (1996) sugieren que es difícil verbalizar los procesos implicados en la tarea TOL, lo que implicaría que debe realizarse algún tipo de ejercicio visuoespacial. Welsh et al. (1995) examinaron el rendimiento en una población no clínica y realizaron una entrevista después de cada ensayo. Los participantes informaron que "visualizaban mentalmente el movimiento de los anillos en las clavijas". Según los autores, esta visualización implicaría el uso de ensayos visuoespaciales para la resolución de la tarea.

No obstante, hay autores y evidencia empírica que está a favor de que la ejecución de la TOL requiere de recursos lingüísticos y no visuoespaciales. Morris et al. (1993) sostienen que en la ejecución de la TOL se evidencia una activación cerebral del hemisferio izquierdo asociada con mecanismos verbales. Además, los déficits en la planificación tienden a estar más asociados con lesiones cerebrales izquierdas (Glosser y Goodglass, 1990; Shallice, 1982), aunque se hayan reportado casos de alteraciones en la capacidad de planificación luego de lesiones del hemisferio derecho (Owen et al., 1990).

Phillips et al. (1999) utilizaron un paradigma de investigación para poner a prueba la hipótesis de la participación de mecanismos verbales y visuoespaciales en la ejecución de la TOL. En un estudio experimental con cuatro condiciones, evaluaron la relación entre la memoria de trabajo verbal (bucle fonológico), la memoria de trabajo visuoespacial (agenda visuoespacial), el ejecutivo central y la ejecución de la TOL. Sus análisis mostraron que la participación de mecanismos visuoespaciales tiene un efecto mayor que los mecanismos verbales sobre la ejecución del TOL.

Uno de los razonamientos que los autores tuvieron en consideración para apoyar este resultado 
fue su idea de que la planificación requerida para la TOL era del tipo on-line y no del tipo pre planning. Según ellos, la ejecución de la TOL requiere mecanismos ejecutivos y visuoespaciales que continuamente permitan establecer el estado pasado reciente, actual y futuro inmediato, más que una planificación completamente preestablecida. Este es el tipo de diferenciación que habían establecido Ward y Allport (1997) entre mental preplanning y on-line planning, aunque ellos apoyaron la idea de que la TOL requería fundamentalmente del primero, y no del segundo, como lo sugieren Phillips et al. (1999).

Un estudio más reciente, realizado por Cheetham, Rahm, Kaller, y Unterrainer (2012), analizó la misma relación entre las habilidades visuoespaciales, las verbales y la ejecución en la ToL y reportaron que las primeras, a diferencia de las segundas, podían predecir significativamente el desempeño en la tarea. Adicionalmente evaluaron la relación entre dos estilos cognitivos (visual "visualizer" y verbal "verbalizer") y la ejecución en la TOL. En este punto no encontró ninguna relación. Según ellos, el funcionamiento neuropsicológico (habilidades cognitivas) tenía más efecto que el estilo cognitivo en la ejecución de la tarea.

\section{Metodología}

\section{Diseño}

Fue un estudio cuantitativo, no experimental, descriptivo, correlacional y transversal. La muestra estuvo conformada por 100 personas con edades entre 10 y 30 años. El objetivo general fue analizar las relaciones evolutivas entre la planificación cognitiva y la memoria de trabajo visuoespacial en un grupo de 100 personas con inteligencia normal y con edades entre los diez (10) y los treinta (30) años. Los criterios de inclusión fueron: sin consumo de sustancias psicoactivas en un periodo inferior o igual a tres (3) meses, sin antecedentes personales o familiares de enfermedad neurológica o psicopatológica diagnosticada, con inteligencia normal de acuerdo con el criterio definido en las Matrices Progresivas de Raven (Raven y Court, 2001): Grado III o intelectualmente promedio en capacidad intelectual, si el centil se encuentra entre los valores 25 y 75 , es decir en el intervalo 26-74.

\section{Procedimiento}

Se reunió un grupo de 100 personas que cumplieron con los criterios de inclusión. A todos los participantes se les aplicó el Raven para garantizar que cumplieran con el criterio de "inteligencia normal/ promedio". La muestra fue dividida de acuerdo con tres rangos de edad: 10 a 15 años, 16 a 20 años y 21 a 30 años. No fue posible garantizar un número equivalente de participantes en cada rango. A cada participante se le aplicó el Subtest de Cubos de Corsi, de la Escala de Memoria de Wechsler III (Wechsler, 2004) y la Torre de Londres. La aplicación estuvo a cargo de estudiantes de último nivel de la carrera de psicología, quienes fueron capacitados y entrenados por un neuropsicólogo (Doctor y Máster en Neuropsicología). Los datos fueron sistematizados y analizados en SPSS versión 22 .

\section{Instrumentos}

Matrices Progresivas de Raven (Raven y Court, 2001): se utilizó para determinar el nivel intelectual. Es un instrumento diseñado para medir la capacidad educativa, mediante la comparación de formas y el razonamiento por analogía con independencia de los conocimientos adquiridos. Utiliza una serie de figuras geométricas abstractas incompletas (matrices). La aplicación y calificación se realizó siguiendo el manual.

Subtest de Cubos de Corsi de la Escala de Memoria de Wechsler III (Wechsler, 2004): se utilizó para evaluar la memoria de trabajo visuoespacial. Es una serie de diez cubos de color azul sobre una plataforma de color blanco. El examinado debe repetir la secuencia que haya realizado el evaluador en orden directo y en orden indirecto. La aplicación y calificación se realizó siguiendo el manual.

Torre de Londres (Shallice, 1982): se utilizó para evaluar la planificación cognitiva. Se siguió el protocolo definido en Krikorian, Bartok y Gay (1994) utilizando las tres esferas de diferentes colores a 
partir de una posición inicial para alcanzar una posición final en una serie de ensayos que variaban en el número de movimientos.

\section{Resultados}

Los análisis de correlación para las variables Memoria de Trabajo Visuoespacial (en adelante Cubos de Corsi) y Planificación Cognitiva (en adelante Torre de Londres) en la muestra completa (10-30 años de edad) indicaron la existencia de una correlación moderada entre estas variables. Este valor en la correlación confirma la hipótesis inicial respecto a la existencia de una asociación entre ambas variables. Este punto se desarrollará en el análisis y la discusión de resultados.

Los análisis de correlación para evaluar la relación evolutiva entre ambas variables también confirmaron la existencia de la asociación, aunque los valores variaron en función del rango de edad seleccionado. Hay que decir que estos rangos no fueron elegidos con base en algún criterio teórico asociado con la psicología evolutiva o la neuropsicología. Los rangos se seleccionaron por conveniencia para poner a prueba la hipótesis de que la correlación entre las variables tiene un curso diferenciado a lo largo del desarrollo cognitivo. Este punto se desarrollará en el análisis y la discusión de resultados.

Otro de los resultados que apoya la hipótesis de una relación evolutiva diferenciada entre ambas variables es el de la prueba de hipótesis para diferencia entre medias de los rangos de edad. Según los resultados, los desempeños en los Cubos de Corsi y en la Torre de Londres en el rango de edad de 10 a 15 años son estadísticamente diferentes de los desempeños en las mismas variables en el rango de edad de 16 a 20 años. Sin embargo, esta diferencia no se observa cuando se comparan los rangos 16-20 y 21-30. Este punto se desarrollará en el análisis y la discusión de resultados.

\section{Resultados para la muestra completa (10 a 30 años de edad)}

\section{Estadísticos descriptivos}

En la tabla 1 se presentan los estadísticos descriptivos de todas las variables para la muestra completa (de 10 a 30 años de edad). Los valores de la media fueron: 18 años para la edad, 10 años de educación, 46,4 puntos en el Test de Matrices Progresivas de Raven, 15,2 puntos en los Cubos de Corsi, y 31,4 puntos en la Torre de Londres.

Tabla 1.

Estadísticos descriptivos de las variables en la muestra completa

\begin{tabular}{lcccc}
\hline \multicolumn{1}{c}{ Variable } & N & Media & Mediana & $\begin{array}{c}\text { Desviación } \\
\text { estándar }\end{array}$ \\
\hline Edad & 100 & 18.0 & 17.0 & 5.5 \\
\hline Años de educación & 100 & 10.1 & 11.0 & 2.4 \\
\hline Raven & 100 & 46.4 & 47.0 & 3.7 \\
\hline Cubos de Corsi & 100 & 15.3 & 15.0 & 4.3 \\
\hline Torre de Londres & 100 & 31.4 & 32.0 & 3.9 \\
\hline
\end{tabular}

\section{Test de normalidad}

Se realizaron los test de normalidad (curtosis, asimetría y Kolmogorov-Smirnov) para las variables Cubos de Corsi y Torre de Londres en la muestra completa. Los resultados se presentan en la tabla 2. De acuerdo con los análisis, ambas variables presentan distribuciones no paramétricas.

Tabla 2 .

Test de normalidad para las variables "Cubos de Corsi" y "Torre de Londres" en la muestra completa

\begin{tabular}{ccccc}
\hline \multicolumn{1}{c}{ Variable } & Asimetría & Curtosis & Kolmogorov-Smirnov & Distribución \\
\hline \multirow{2}{*}{ Cubos de Corsi } & $\begin{array}{l}\text { z-value }=0.714 \\
p \text {-value }=0.475\end{array}$ & $\begin{array}{l}\text { z-value }=0.016 \\
p \text {-value }=0.987\end{array}$ & $\begin{array}{l}D(100)=0.102 \\
p \text {-value }=0.012\end{array}$ & No paramétrica \\
\hline \multirow{2}{*}{ Torre de Londres } & $\begin{array}{l}\text { z-value }=4.105 \\
p \text {-value }<0.001\end{array}$ & $\begin{array}{l}\text { z-value }=2.328 \\
p \text {-value }=0.020\end{array}$ & $\begin{array}{l}D(100)=0.132 \\
p \text {-value }<0.001\end{array}$ & No paramétrica \\
\hline
\end{tabular}




\section{Coeficiente de correlación}

Debido a que las dos variables tienen una distribución no paramétrica, se realizó un análisis de correlación no paramétrico mediante el coeficiente de correlación de Spearman ( $\rho$ ) en la muestra completa para las variables Cubos de Corsi y Torre de Londres. Los resultados se presentan en la tabla 3. El valor de la correlación fue moderado $(\rho=0.43)$. El coeficiente de determinación, que corresponde al porcentaje de la variabilidad de los datos que se explica por la asociación entre las dos variables, es de 0.18 , lo cual indica que existe hasta un $18 \%$ de varianza compartida.

Tabla 3.

Coeficiente de correlación de Spearman ( $\rho$ ) para las variables "Cubos de Corsi" y "Torre de Londres" en la muestra completa

\begin{tabular}{lcc}
\hline & Cubos de Corsi & Torre de Londres \\
\hline Cubos de Corsi & 1.0 & \\
\hline Torre de Londres & $0.43^{*}$ & 1.0 \\
\hline${ }^{*} p$-value $<0.001$ & & \\
\hline
\end{tabular}

Estos análisis de correlaciones corresponden a la muestra completa, que incluye todo el rango de edad evaluado (de 10 a 30 años). Sin embargo, como el objetivo del estudio es analizar las relaciones evolutivas, es necesario establecer el perfil evolutivo en tres grupos de edad y realizar los análisis estadísticos en estos mismos grupos.

\section{Resultados para los rangos de edad (10 a 16 años, 16 a 20 años, 21 a 30 años)}

\section{Estadísticos descriptivos}

En la tabla 4 se presentan los estadísticos descriptivos de todas las variables para el rango de edad de 10 a 15 años. Este rango estuvo conformado por 35 personas. Los valores de la media fueron: 12,5 años de edad, 7,5 años de educación, 45,4 puntos en el Test de Matrices Progresivas de Raven, 14,2 puntos en los Cubos de Corsi, y 29,9 puntos en la Torre de Londres. En la misma tabla se presentan los estadísticos descriptivos de todas las variables para el rango de edad de 16 a 20 años. Este rango estuvo conformado por 37 personas. Los valores de la media fueron: 12,5 años de edad, 7,5 años de educación, 45,4 puntos en el Test de Matrices Progresivas de Raven, 14,2 puntos en los Cubos de Corsi, y 29,9 puntos en la Torre de Londres. Finalmente, se presentan los estadísticos descriptivos de todas las variables para el rango de edad de 21 a 30 años. Este rango estuvo conformado por 28 personas. Los valores de la media fueron: 25,1 años de edad, 11,8 años de educación, 45,7 puntos en el Test de Matrices Progresivas de Raven, 13,9 puntos en los Cubos de Corsi, y 30,4 puntos en la Torre de Londres.

Tabla 4.

Estadísticos descriptivos de las variables por rangos de edad

\begin{tabular}{lcccc}
\hline Variable & N & Media & Mediana & $\begin{array}{c}\text { Desviación } \\
\text { Estándar }\end{array}$ \\
\hline Rango de edad 10 a & 15 años & & \\
\hline Edad & 35 & 12.5 & 13.0 & 1.7 \\
\hline Años de educación & 35 & 7.5 & 8.0 & 1.6 \\
\hline Raven & 35 & 45.4 & 47.0 & 4.0 \\
\hline Cubos de Corsi & 35 & 14.2 & 15.0 & 3.2 \\
\hline Torre de Londres & 35 & 29.9 & 31.0 & 4.3 \\
\hline Rango de edad 16 a 20 & años & & \\
\hline Edad & 37 & 17.8 & 18.0 & 1.2 \\
\hline Años de educación & 37 & 11.3 & 11.0 & 0.5 \\
\hline Raven & 37 & 47.6 & 48.0 & 3.4 \\
\hline Cubos de Corsi & 37 & 17.3 & 17.0 & 4.5 \\
\hline Torre de Londres & 37 & 33.4 & 35 & 2.7 \\
\hline Rango de edad 21 & a 30 & años & & \\
\hline Edad & 28 & 25.1 & 25.0 & 3.0 \\
\hline Años de educación & 28 & 11.8 & 11.0 & 1.7 \\
\hline Raven & 28 & 45.7 & 46.5 & 3.2 \\
\hline Cubos de Corsi & 28 & 13.9 & 15.0 & 3.8 \\
\hline Torre de Londres & 28 & 30.4 & 31.0 & 3.9 \\
\hline
\end{tabular}

\section{Test de normalidad}

Se realizaron los test de normalidad (curtosis, asimetría y Shapiro-Wilk) para las variables Cubos de Corsi y Torre de Londres en el rango de edad de 10 a 15 años. De acuerdo con los análisis, la variable Cubos de Corsi presenta una distribución paramétrica mientras que la variable Torre de Londres presenta una distribución no paramétrica. Se realizaron los test de normalidad (curtosis, asimetría 
Tabla 5.

Test de normalidad para las variables "Cubos de Corsi" y "Torre de Londres" por rangos de edad

\begin{tabular}{|c|c|c|c|c|}
\hline Variable & Asimetría & Curtosis & Shapiro-Wilk & Distribución \\
\hline \multicolumn{5}{|c|}{ Rango de edad 10 a 15 años } \\
\hline Cubos de Corsi & $\begin{array}{l}z \text {-value }=1.406 \\
p \text {-value }=0.160\end{array}$ & $\begin{array}{l}z \text {-value }=-0.077 \\
p \text {-value }=0.938\end{array}$ & $\begin{array}{l}W=0.951 \\
p \text {-value }=0.122\end{array}$ & Paramétrica \\
\hline Torre de Londres & $\begin{array}{l}z \text {-value }=-1.909 \\
p \text {-value }=0.056\end{array}$ & $\begin{array}{l}z \text {-value }=0.412 \\
p \text {-value }=0.681\end{array}$ & $\begin{array}{l}W=0.929 \\
p \text {-value }=0.026\end{array}$ & No paramétrica \\
\hline \multicolumn{5}{|c|}{ Rango de edad 16 a 20 años } \\
\hline Cubos de Corsi & $\begin{array}{l}z \text {-value }=2.334 \\
p \text {-value }=0.020\end{array}$ & $\begin{array}{l}z \text {-value }=1.639 \\
p \text {-value }=0.101\end{array}$ & $\begin{array}{l}W=0.905 \\
p \text {-value }=0.004\end{array}$ & No paramétrica \\
\hline Torre de Londres & $\begin{array}{l}z \text {-value }=-2.090 \\
p \text {-value }=0.037\end{array}$ & $\begin{array}{l}z \text {-value }=-0.087 \\
p \text {-value }=0.930\end{array}$ & $\begin{array}{l}W=0.838 \\
p \text {-value }<0.001\end{array}$ & No paramétrica \\
\hline \multicolumn{5}{|c|}{ Rango de edad 21 a 30 años } \\
\hline Cubos de Corsi & $\begin{array}{l}z \text {-value }=-1.255 \\
p \text {-value }=0.211\end{array}$ & $\begin{array}{l}z \text {-value }=-0.507 \\
p \text {-value }=0.612\end{array}$ & $\begin{array}{l}W=0.939 \\
p \text {-value }=0.105\end{array}$ & Paramétrica \\
\hline Torre de Londres & $\begin{array}{l}z \text {-value }=-3.205 \\
p \text {-value }=0.001\end{array}$ & $\begin{array}{l}z \text {-value }=2.618 \\
p \text {-value }=0.009\end{array}$ & $\begin{array}{l}W=0.839 \\
p \text {-value }<0.001\end{array}$ & No paramétrica \\
\hline
\end{tabular}

y Shapiro-Wilk) para las variables Cubos de Corsi y Torre de Londres en el rango de edad de 16 a 20 años. De acuerdo con los análisis, ambas variables presentan una distribución no paramétrica. Finalmente, se realizaron los test de normalidad (curtosis, asimetría y Shapiro-Wilk) para las variables Cubos de Corsi y Torre de Londres en el rango de edad de 21 a 30 años. De acuerdo con los análisis, la variable Cubos de Corsi presenta una distribución paramétrica, mientras que la variable “Torre de Londres" presenta una distribución no paramétrica. Todos los resultados se presentan en la tabla 5.

\section{Coeficiente de correlación}

Para el rango de edad de 10 a 16 años, y debido a que una de las dos variables tiene una distribución no paramétrica, se realizó un análisis de correlación no paramétrico mediante el coeficiente de correlación de Spearman $(\rho)$. El valor de la correlación fue moderado $(\rho=0.44)$. El coeficiente de determinación, que corresponde al porcentaje de la variabilidad de los datos que se explica por la asociación entre las dos variables, es de 0.18 , lo cual indica que existe hasta un $18 \%$ de varianza compartida. Para el rango de edad 16 a 21 años, y debido igualmente a que las dos variables tienen una distribución no paramétrica, se realizó un análisis de correlación no paramétrico mediante el coeficiente de correlación de Spearman $(\rho)$. El valor de la correlación fue bajo $(\rho=0.28)$. El coeficiente de determinación, que corresponde al porcentaje de la variabilidad de los datos que se explica por la asociación entre las dos variables, es de 0.07 , lo cual indica que existe hasta un 7 $\%$ de varianza compartida. Finalmente, para el rango de edad de 21 a 30 años, y debido a que una de las dos variables tiene una distribución no paramétrica, se realizó un análisis de correlación no paramétrico mediante el coeficiente de correlación de Spearman $(\rho)$. El valor de la correlación fue moderado $(\rho=0.32$ ). El coeficiente de determinación, que corresponde al porcentaje de la variabilidad de los datos que se explica por la asociación entre las dos variables, es de 0.10 , lo cual indica que existe hasta un $10 \%$ de varianza compartida. Todos los resultados se presentan en la tabla 6. 
Tabla 6.

Coeficiente de correlación de Spearman ( $\rho$ ) para las variables "Cubos de Corsi" y "Torre de Londres" por rangos

\begin{tabular}{|c|c|c|}
\hline & $\begin{array}{c}\text { Cubos de } \\
\text { Corsi }\end{array}$ & Torre de Londres \\
\hline \multicolumn{3}{|c|}{ Rango de edad 10 a 15 años } \\
\hline Cubos de Corsi & 1.0 & \\
\hline Torre de Londres & $0.44^{*}$ & 1.0 \\
\hline \multicolumn{3}{|c|}{ Rango de edad 16 a 20 años } \\
\hline Cubos de Corsi & 1.0 & \\
\hline Torre de Londres & $0.28^{*}$ & 1.0 \\
\hline \multicolumn{3}{|c|}{ Rango de edad 21 a 30 años } \\
\hline Cubos de Corsi & 1.0 & \\
\hline Torre de Londres & $0.32^{*}$ & 1.0 \\
\hline two-tailed $p$ valu & 0.008 & \\
\hline
\end{tabular}

\section{Comparativo entre los rangos de edad}

\section{Prueba de hipótesis para diferencias entre medias}

Tabla 7.

Prueba de hipótesis (Mann-Whitney) para la diferencia entre medias entre los rangos de edad

\begin{tabular}{lll}
\hline & \multicolumn{1}{c}{$\begin{array}{c}\text { Rango } \\
(10-15) /(16-20)\end{array}$} & $\begin{array}{c}\text { Rango } \\
(16-20) /(21-30)\end{array}$ \\
\hline \multirow{3}{*}{ Cubos de Corsi } & $\begin{array}{l}\text { z-score }=-3.463 \\
p<0.001^{*}\end{array}$ & $\begin{array}{l}\text { z-score }=-3.014 \\
p=0.003^{*}\end{array}$ \\
\hline \multirow{2}{*}{ Torre de Londres } & $\begin{array}{l}\text { z-score }=-3.752 \\
p<0.001^{*}\end{array}$ & $\begin{array}{l}\text { z-score }=-3.406 \\
p<0.001^{*}\end{array}$ \\
\hline
\end{tabular}

* Statistically siginificant difference (2-tailed)

\section{Análisis y discusión de resultados}

Los resultados respaldan las dos hipótesis sobre las cuales se elaboró el proyecto: 1) existe una relación entre la memoria de trabajo visual y la planificación cognitiva, y 2 ) esta relación varía en función del rango de edad en personas sanas. La relación entre la memoria de trabajo visual y la planificación cognitiva logra establecerse mediante un análisis de correlación en personas sanas y con un nivel normal de inteligencia. La correlación entre estas variables fue de grado medio $(0.43)$ y estadísticamente significativa ( $p$-value $<0.001$ ) en la muestra completa (rango de 10 a 30 años).

Este valor indica que sí existe una asociación entre estas dos funciones ejecutivas (memoria de trabajo visual y la planificación cognitiva) y que existe hasta un $18 \%$ de varianza compartida entre ambas variables. Este $18 \%$ no debería parecer bajo, pues en ningún momento se ha afirmado que la planificación cognitiva dependa exclusivamente de la memoria de trabajo visual. La hipótesis que se planteó, a partir de inferencias teóricas y el respaldo empírico, era la existencia de la asociación, más no la dependencia exclusiva de una variable en relación con la otra.

Albrecht y Ragni (2014) desarrollaron un modelo para explicar la ejecución en la Torre de Londres denominado ACT-R (Spatial Planning), es un modelo de arquitectura cognitiva compuesto por tres módulos: un módulo para procesamiento de información visual (módulo visual), un módulo para representar la información relacionada con el objetivo (módulo de objetivo), y un módulo para almacenar y recuperar información sobre memoria declarativa (módulo declarativo). Según lo que puede inferirse del modelo, la planificación ocurre on-line y depende de las constricciones externas que presente la configuración particular de los anillos en las clavijas en un momento específico de la ejecución. Además, el procesamiento visuoespacial y la memoria de trabajo son componentes esenciales dentro del funcionamiento del sistema de planificación.

La correlación encontrada en este estudio es consistente con la idea de una asociación (¿mecanismo cognitivo subyacente?) entre la planificación cognitiva y la memoria de trabajo visuoespacial. Este grado de correlación coincide con la propuesta que presenta el modelo ACT-R: la memoria de trabajo visuoespacial podría estar relacionada tanto con el módulo visual como con el módulo declarativo. Es decir, si bien es cierto que la correlación existe, el grado de correlación no debería ser más alto porque se espera que la planificación, como proceso, no se limite a uno o dos mecanismos sino que, por 
ser un proceso ejecutivo, exista un número mayor de mecanismos que le subyazcan.

Lo relevante de la correlación no es solo la dimensión "visuoespacial" sino también el hecho de que se evaluó en la modalidad de memoria de trabajo. Los mecanismos visuoespaciales de la memoria de trabajo involucrados en la planificación indican que se requiere de información visuoespacial y que esta información se utiliza momento a momento en la ejecución, tal y como lo sostienen Phillips et al. (2001), quienes reportaron que la ejecución del TOL puede realizarse a modo de mental preplanning, con una limitación de hasta siete movimientos (o dos subobjetivos), sin que sea posible planear más de nueve movimientos (tres o cuatro subobjetivos), con lo cual no es posible realizar una previa planificación completa.

A partir de este hallazgo, los autores afirman que el mental preplanning no es el determinante para la ejecución de la TOL, sino que, por el contrario, la planificación on-line es sobre la que descansa el desempeño efectivo en la tarea. Así, sostienen que las "decisiones realizadas durante la planificación pueden estar influidas por los cambios momento a momento en el estado de los atributos relevantes" (Philips et al, 2001, p.17). Aquí habría que añadir, aunque esto no aparece en las conclusiones de su estudio, que los atributos relevantes son, en buena medida, visuoespaciales.

La participación, confirmada con la correlación, de mecanismos visuoespaciales en la ejecución de la TOL también apoya los hallazgos de Welsh et al. (1995) en los que los participantes de estudio informaron que "visualizaban mentalmente el movimiento de los anillos en las clavijas" (p.79). Este tipo de "visualización mental" momento a momento requiere de la agenda visuoespacial de la memoria de trabajo. Esta agenda es la responsable de mantener en la memoria inmediata la información visual y espacial necesaria para ser utilizada online por otros mecanismos cognitivos.

Se ha sugerido que la memoria de trabajo visuoespacial funciona en codependencia con otro tipo de memoria que almacena secuencias de acciones en un código kinestésico (Smyth y Pendleton,
1989). De acuerdo con Logie (1995), este código, junto con el componente visuoespacial, origina el escriba interior (inner scribe), que tienen como función mantener la información sobre la secuencia de movimientos, de manera que, al evaluar la memoria de trabajo visuoespacial, también se está evaluando, indirectamente el código kinestésico y el escriba interior, y ambos respaldan la hipótesis de la ejecución de la TOL requiere recursos visuales, espaciales y secuenciales para lograr lo que Albrecht y Ragni (2014) denominan como planificación espacial.

Los análisis evolutivos por rangos de edad (10 a 15 años; 16 a 20 años; 21 a 30 años) mostraron que el grado de la correlación entre la memoria de trabajo visuoespacial y la planificación cognitiva va disminuyendo con la edad: $\rho$ de 0,44 para el rango 10 a 15 años, $\rho$ de 0,28 para el rango de 16 a 20 años y $\rho$ de 0,32 para el rango 21 a 30 años. Esta tendencia, aunque no es perfecta, no puede más que insinuarse porque el número de participantes por rango fue relativamente bajo (aproximadamente 30 , en promedio). Lo que sí resulta interesante estadísticamente fue la existencia de diferencias significativas en las puntuaciones medias en los Cubos de Corsi y la Torre de Londres entre los rangos (tabla 7), la puntuación significativamente mayor del rango intermedio y la puntuación significativamente menor del último rango.

Tanto la memoria de trabajo visuoespacial como la planificación cognitiva están asociadas con el funcionamiento de la corteza prefrontal (Courtney, Petit, Haxby \& Ungerleider, 1998; Tsujimoto, 2008) que es la región cerebral que más tiempo tarda en madurar (milelinización) durante la ontogenia (Fuster, 2002). La materia gris prefrontal aumenta volumétricamente después del nacimiento y alcanza un máximo entre los 4 y los 12 años, momento a partir del cual comienza a disminuir gradualmente (Pfefferbaum, Mathalon, Sullivan, Rawles, Zipursky \& Lim, 1994; Giedd, Blumenthal, Jeffries, Castellanos, Liu, Zijdenbos, Paus, Evans \& Rapoport, 1999), pero la maduración completa y la conectividad se establece en la adultez al inicio de la tercera década de la vida. De manera que es posible esperar variaciones en el funcionamiento cognitivo de estas funciones en relación con la edad. 
En esta lógica, Gathercole, Pickering, Ambridge \& Wearing (2004) reportaron un aumento lineal en el funcionamiento de la memoria de trabajo visuoespacial (evaluada mediante Cubos de Corsi) en un rango de edad entre 4 y 15 años en niños y adolescentes sanos. Este estudio respalda los hallazgos previos reportados por Pickering (2001) en relación con un aumento progresivo en el funcionamiento de la memoria de trabajo visuoespacial a medida que aumenta la edad. El desempeño en la planificación cognitiva, evaluada mediante la ToL, también mejora con la edad. Algunos estudios reportan que el nivel de la ejecución mejora desde la infancia hasta la adolescencia (Krikorian, Bartok \& Gay, 1994; Luciana \& Nelson, 1998, 2002).

Resulta difícil explicar por qué, entonces, en este estudio no se encontró este aumento lineal en el funcionamiento de la memoria de trabajo visuoespacial ni en la planificación cognitiva. Además, si se tiene en consideración que la inteligencia fue una variable que se controló desde el inicio (todos los participantes tenían inteligencia normal según la puntuación establecida en el Raven) no podría conjeturarse que estas diferencias se deban a factores intelectuales. De hecho, resulta contradictorio que los resultados del funcionamiento ejecutivo en el tercer rango, el de adultos jóvenes con edades entre 21 y 30 años, haya sido el de menor rendimiento, según la lógica antes mencionada.

Una explicación plausible puede estar asociada con las características socioculturales y socioeconómicas de una parte de los participantes que conformaron este último rango de edad. El $35 \%$ de los participantes en ese rango de edad fueron evaluados en la ciudad de Montería, una región del país diferente a la región donde está la ciudad de Medellín, que fue donde se realizó el estudio. El estrato socioeconómico y las variables socioculturales no fueron controlados. De manera que es posible que, aunque estas personas de la ciudad de Montería cumplieron con el criterio de inteligencia normal, existiesen factores socioculturales que influyeron sobre el desempeño en estas funciones ejecutivas, como ha sido sugerido por algunos estudios (Carlson, 2009; Lewis, Koyasu, Oh, Ogawa, Short y Huang, 2009).

\section{Conclusiones}

El estudio encontró una correlación media y estadísticamente significativa entre la memoria de trabajo visuoespacial y la planificación cognitiva. Esta correlación era esperable puesto que ambas funciones forman parte del cúmulo teórico de las funciones ejecutivas. El aporte del estudio radica más en haber explorado esta relación en tres grupos evolutivos, en los que hubo correlaciones estadísticamente significativas. Sin embargo, no se encontró la tendencia linean hacia un aumento en el funcionamiento ejecutivo con el aumento de la edad. Los análisis reportaron un mejor funcionamiento y una mayor correlación entre las variables en el grupo más joven (10 a 15 años), y diferencias estadísticamente significativas entre los tres grupos de edad (10 a 15 años, 16 a 20 años, 21 a 30 años). Pese a que se controló la habilidad intelectual general, mediante un criterio de exclusión definido a partir del puntaje en las matrices progresivas de Raven, es posible que existan otros factores no intelectuales (socioeconómicos y socioculturales) que hayan podido afectar el rendimiento cognitivo en los dos test de función ejecutiva que se utilizaron. Esta hipótesis se ha planteado para intentar comprender por qué el último grupo (21 a 30 años) obtuvo el menor de los rendimientos.

\section{Referencias}

Albrecht, R. \& Ragni, M. (2014). Spatial Planning: An ACT-R model for the Tower of London Task. In Spatial Cognition Ix (pp. 222-236). Cham: Springer International Publishing.

Carlson, S.M. (2009). Social origins of executive function development. New Directions in Child and Adolescent Development, 123, 87-97. Retrieved from https://www.ncbi.nlm.nih.gov/ pubmed/19306276

Cheetham, J.M., Rahm, B., Kaller, C.P., y Unterrainer, J.M. (2012). Visuospatial over verbal demands in predicting Tower of London planning tasks. British Journal of Psychology, 103(1), 98-116. Retrieved from https://www.ncbi.nlm.nih.gov/ pubmed/22229777 
Cohen, G. (1996). Memory in the real world (2nd Edn.). Hove, UK: Psychology Press.

Courtney, S.M., Petit, L., Maisog, J.M., Ungerleider, L.G. \& Haxby, J. V. (1998). An area specialized for spatial working memory in human frontal cortex. Science, 279(5355), 1347-51. Retrieved from https://www.ncbi.nlm.nih.gov/ pubmed/9478894

Fuster, J. M. (2002). Frontal lobe and cognitive development. Journal of Neurocitology, 31, 373385. Retrieved from https://www.ncbi.nlm.nih. gov/pubmed/12815254

Gathercole, S.E., Pickering, S.J., Ambridge, B. \& Wearing, H. (2004). The structure of working memory from 4 to 15 years of age. Developmental Psychology, 40, 177-190. Retrieved from https://www.ncbi.nlm.nih.gov/ pubmed/14979759

Giedd, J.N., Blumenthal, J., Jeffries, N.O., Castellanos, F.X., Liu, H., Zijdenbos, A., Paus, T., Evans, A.C \& Rapoport, J. L. (1999). Brain development during childhood and adolescence: a longitudinal MRI study. Nature Neuroscience, 2, 861-863. Retrieved from http://www.nature.com/neuro/ journal/v2/n10/abs/nn1099_861.html

Glosser, G. \& Goodglass, H. (1990). Disorders in executive control functions among aphasic and other brain damaged patients. Journal of Clinical and Experimental Neuropsychology, 12, 485-501. Retrieved from https://www.ncbi.nlm. nih.gov/pubmed/1698809

Joyce, E. M. \& Robbins, T.W. (1991). Frontal lobe function in Korsakoff and non-Korsakoff alcoholics: planning and spatial working memory. Neuropsychologia, 29, 709-723. Retrieved from https://www.ncbi.nlm.nih.gov/pubmed/ 1944873

Krikorian, R., Bartok, J. \& Gay, N. (1994). Tower of London procedure: A standard method and developmental data. Journal of Clinical and Experimental Neuropsychology, 16, 840-850. Retrieved from https://www.ncbi.nlm.nih.gov/ pubmed/7890819
Lewis, C., Koyasu, M., Oh, S., Ogawa, A., Short, B. \& Huang, Z. (2009). Culture, executive function, and social understanding. New Directions for Children Adolescent Development, 123, 69-85. Retrieved from https://www.ncbi.nlm.nih.gov/ pubmed/19306275

Logie, R.H. (1995). Visuo-spatial Working Memory. Hove: Lawrence Erlbaum.

Luciana, M. y Nelson, C.A. (1998). The functional emergence of prefrontally-guided memory systems in four- to eight-year-old children. Neuropsychologia, 36, 272-293. Retrieved from https://www.ncbi.nlm.nih.gov/ pubmed/9622192

Luciana, M., Collins, P.F., Olson, E.A., y Schissel, A.M. (2009). Tower of London performance in healthy adolescents: The development of planning skills and associations with self-reported inattention and impulsivity. Developmental Neuropsychology, 34(4), 461475. Retrieved from https://www.ncbi.nlm.nih. gov/pubmed/20183711

Luciana, M., y Nelson, C.A. (2002). Assessment of neuropsychological function through use of the Cambridge Neuropsychological Testing Automated Battery: performance in 4- to 12-yearold children. Developmental Neuropsychology, 22, 595-624. Retrieved from https://www.ncbi. nlm.nih.gov/pubmed/12661972

Morris, R.G., Ahmed, S., Syed, G.M.S., y Toone, B.K. (1993). Neural correlates of planning ability: Frontal lobe activation during the Tower of London test. Neuropsychologia. 21(12), 13671378. Retrieved from http://www.sciencedirect. com/science/article/pii/0028393293901048

Owen, A.M., Downes, J.D., Sahakian, B.J., Polkey, C.E., y Robbins, T.W. (1990). Planning and spatial working memory following frontal lobe lesions in man. Neuropsychologia, 28, 1021-1034. Retrieved from https://www.ncbi.nlm.nih.gov/ pubmed/2267054

Pfefferbaum, A., Mathalon, D.H., Sullivan, E.V., Rawles, J.M., Zipursky, R.B. \& Lim, K. O. (1994). 
A quantitative magnetic resonance imaging study of changes in brain morphology from infancy to late adulthood. Archives of Neurology, 51, 874-887. Retrieved from https://www.ncbi. nlm.nih.gov/pubmed/8080387

Phillips, L.H., Wynn, V., Gilhooly, K.J., Della Sala, S. \& Logie, R.H. (1999). The role of memory in the Tower of London task. Memory, 7(2), 20931. Retrieved from https://www.ncbi.nlm.nih. gov/pubmed/10645380

Pickering, S. J. (2001). The development of visuospatial working memory. Memory, 9(4-6), 42332. Retrieved from https://www.ncbi.nlm.nih. gov/pubmed/11594361

Raven, J. \& Court, J. (2001). Raven Matrices Progresivas ( 3 ed.). Madrid: TEA Ediciones S.A.

Shallice, T. (1982). Specific impairments of planning. Philosophical Transactions of the Royal Society of London B, 298, 199-209. Retrieved from https://www.ncbi.nlm.nih.gov/ pubmed/6125971

Smyth, M.M., \& Pendleton, L.R. (1989). Working memory for movements. Quarterly Journal of Experimental Psychology, 41A, 235-250. Retrieved from http://www.tandfonline.com/ doi/abs/10.1080/14640748908402363?journal Code=pqja20
Temple, C.M., Carney, R.A., y Mullarkey, S. (1996). Frontal lobe function and executive skills in children with Turner's syndrome. Developmental Neuropsychology, 12, 343-363. Retrieved from http://www.tandfonline.com/doi/abs/10.108 0/87565649609540657? journalCode=hdvn20

Tsujimoto, S., (2008). The prefrontal cortex: Functional neural development during early childhood. The Neuroscientist, 14, 345-358. Retrieved from https://www.ncbi.nlm.nih.gov/ pubmed/18467667

Ward, G., \& Allport, A. (1997). Planning and problemsolving using the 5-disc Tower of London task. Quarterly Journal of Experimental Psychology, 50, 49-78. Retrieved from http://www.tandfonline.com/doi/abs/10.1080/713755681

Wechsler, D. (2004). Escala de Memoria de Wechsler III: Manual de aplicación. Madrid: TEA Ediciones S.A.

Welsh, M.C., Cicerello, A., Cuneo, K. \& Brennan, M. (1995). Error and temporal patterns in Tower of Hanoi performance: Cognitive mechanisms and individual differences. Journal of General Psychology, 122, 69-81. Retrieved from http:// www.tandfonline.com/doi/abs/10.1080/002213 09.1995 .9921223 